\title{
¿Debe el historiador de las ciencias convertirse en contemporáneo de los científicos de los que habla?
}

\section{Must the Historian of Science Become a Contemporary of the Scientists He Talks About?}

\section{Hélène Metzger}

Traducido por

Juan Felipe Guevara Aristizabal Universidad Nacional Autónoma de México

Violeta Aréchiga Córdova Universidad Autónoma Metropolitana-Cuajimalpa

\section{Presentación}

Hélène Emilie Bruhl nació el 26 de agosto de 1889 cerca de París. Estudió cristalografía en la Sorbona donde obtuvo su diploma de estudios superiores en el año 1912, el cual, a diferencia de un título de licenciatura, no le permitió acceder a un doctorado de Estado, requisito fundamental para comenzar una carrera como académica universitaria. Al año siguiente, se casó con Paul Metzger. Desafortunadamente, su esposo murió poco más de un año después en los combates de la primera gran guerra. A partir de 1914, Hélène Metzger se dedicó de lleno a la historia de las ciencias, cuyo primer logro sería el libro La génesis de la ciencia de los cristales. Su pensión de viuda y la pequeña fortuna con la que contaba le permitieron llevar una vida investigativa independiente, al margen de las instituciones académicas francesas. Sin embargo, esto también le costó el reconocimiento de sus pares, quienes la consideraban como una aficionada. Sería en 1926, gracias a la publicación de Los conceptos científicos, que el aislamiento institucional e intelectual 
llegaría a su fin. A partir de entonces comenzaron una serie de colaboraciones importantes para su trayectoria intelectual: por un lado, con la revista de historia de las ciencias Isis, así como con el Centro de Síntesis, y, por otro, con el Comité Internacional de Historia de las Ciencias, del cual se haría miembro activo a partir de 1928. De igual manera, formó parte del Grupo Francés de Historiadores de las Ciencias desde sus comienzos, lo cual la llevaría a organizar y participar en numerosos congresos del tema. Hubo uno particularmente importante en su biografía: el Congreso de Historia de las Ciencias de 1934 que se celebraría en Berlín. No obstante, ante el ascenso al poder de Hitler y el partido Nacional Socialista, Metzger le escribe a algunos de sus colegas acerca de sus preocupaciones en relación a la comunidad judía. Su voz fue escuchada y gracias a su iniciativa, el congreso cambió de sede y tuvo lugar en España. Durante la última etapa de su vida, la guerra, la ocupación alemana en Francia y la deportación marcaron sus días. En 1941 se mudó a Lyon donde, además, se inscribió en la lista oficial de judíos. A pesar de la insistencia de amigos y colegas, Metzger se rehusó a vivir en la clandestinidad. Durante su tiempo en Lyon se integró al Despacho de Estudios Judíos, grupo en el cual se mantendría activa hasta su arresto en febrero de 1944. En marzo del mismo año fue deportada a Auschwitz. Se desconoce si llegó con vida al campo de concentración o si murió en una cámara de gas a su llegada. ${ }^{1}$

El texto que presentamos a continuación constituye la primera traducción al español de alguna parte de la obra de Hélène Metzger. En este caso, se trata de la traducción del artículo "L'historien des sciences, doit-il se faire le contemporain des savants dont il parle?”, publicado en 1933 en el número 15 de la revista Archeion, que fuera en su momento el órgano de difusión de la Academia Internacional de Historia de las Ciencias. Aunque el tema del escrito es eminentemente histórico/ historiográfico, su bagaje filosófico no deja de ser por ello relevante, en especial si lo pensamos en el contexto de la epistemología francesa y sus innegables lazos con las historia. Las ideas que desarrolla y discute Metzger a lo largo de su texto tienen ecos de otras discusiones filosóficas, tales como aquellas concernientes a la hermenéutica durante el siglo XIX en Alemania, o las reflexiones en torno a la naturaleza de las ciencias del espíritu o de la cultura, así denominadas por Wilhelm Dilthey y Heinrich Rickert, respectivamente. Podríamos afirmar, no obstante, que el tema más acuciante del texto tiene que ver con el talante anacrónico de la labor histórica, idea que iba a contracorriente de la dominante escuela francesa de los Anales,

${ }^{1}$ A las personas interesadas en saber más acerca de la vida de Hélène Metzger, les recomendamos la lectura de Freudenthal (1988), Chimisso $(2001,2019)$ y Chimisso y Freudenthal (2003). 
y de la cual se derivan reflexiones importantes para una consideración filosófica de la historia.

Con respecto a la traducción, quisiéramos ofrecer un par de aclaraciones. La palabra savants, que aparece desde el título del texto, la hemos traducido de tres maneras distintas. En primer lugar, y la más empleada, como hombres de ciencia. Otro equivalente usado es cientificos cuando aparece en la misma oración con historiadores de la ciencia, para evitar la redundancia de los términos compuestos. Por último, también la traducimos como sabios, la forma más literal, cuando el contexto no implica una relación directa con la ciencia. Asimismo, hemos mantenido para los sustantivos y adjetivos la forma masculina dominante en el texto francés. El escrito de Metzger, por otro lado, tiene un tono oral, el cual se entrevé tanto en la construcción de las frases como en el uso constante del punto y coma. Hemos modificado en numerosas ocasiones la puntuación del texto por cuestiones de estilo.

Pese a que desde la década de 1980 su trabajo ha atraído de manera creciente la atención de algunos sectores académicos, su obra sigue sin ser traducida, no sólo en español sino también en inglés. De hecho, la primera monografía dedicada a su obra apareció apenas este ańo (ver Chimisso, 2019). Esperamos que este esfuerzo sea el inicio de una empresa para subsanar esta carencia.

\section{Juan Felipe Guevara Aristizabal y Violeta Aréchiga Córdova}

\section{Referencias}

Chimisso, C. (2001). "Hélène Metzger: The History of Science between the Study of Mentalities and Total History". Studies in History and Philosophy of Science Part A, 32(2), 203-241. doi: https://doi.org/10.1016/S00393681(01)00006-1

Chimisso, C. (2019). Hélène Metzger: Historian and Historiographer of the Sciences. Oxon: Routledge.

Chimisso, C.; y Freudenthal, G. (2003). "A Mind of Her Own: Hélène Metzger to Émile Meyerson, 1933”. Isis, 94(3), 477-491. doi: https://doi. org/10.1086/380655

Freudenthal, G. (1988). "Hélène Metzger: Eléments de biographie". Corpus. Revue de philosophie, 8/9, 197-208. 


\section{¿Debe el historiador de las ciencias convertirse en contemporáneo de los científicos de los que habla?}

Les invito hoy a discutir un tema que me interesa sobremanera; quisiera examinar con ustedes la siguiente pregunta: "¿Debe el historiador de las ciencias tratar de convertirse en contemporáneo de los científicos cuyas teorías expone?”. A esta pregunta metodológica, con la cual me he topado desde que comencé el estudio de la evolución del pensamiento científico, he tenido ya oportunidad de responder que si el historiador no quería o no sabía leer las obras de antaño como las leían sus primeros lectores, se arriesgaba a malinterpretarlas y, por tanto, no sólo hacía que se malinterpretaran los trabajos de nuestros predecesores lejanos, sino que también alteraba la perspectiva del progreso mismo de la ciencia. Esta respuesta no fue aceptada por ninguno de los interesados en el método histórico. En ocasiones, me han reprochado por haberme rehusado a juzgar la doctrina de los siglos pasados a la luz de nuestras teorías actuales. Se ha dicho que era justo aplaudir a ciertos químicos de antańo por haber pensado sobre un punto determinado justo lo que pensamos actualmente. Se ha dicho que se les podría reprochar a ciertos químicos de antaño el haberse alejado de cierta manera de ver que se ha convertido en la nuestra. Y como en efecto muchos historiadores de las ciencias, que no se han tomado la pena de reflexionar sobre la crítica histórica, han hecho (después de haber obtenido un conocimiento superficial de los textos) que sus exposiciones se asemejen a un palmarés de premios - refiriéndose de manera constante a una cierta concepción positivista de la ciencia, o a cierta doctrina admitida al momento de publicar sus recuentos-, se darán cuenta que tengo que justificar mi punto de vista.

Como pueden ver, el problema sobre el cual les pido que arrojen la luz de una franca discusión es uno de los más importantes, quizás incluso el problema sumo del método de la historia de las ciencias, pues de la solución escogida puede depender toda nuestra concepción del pasado de la humanidad, del papel del pensamiento humano, de la experiencia, del empirismo positivo o de la inspiración metafísica en la formación misma de la ciencia. De manera recíproca, me responderán ustedes que de nuestra concepción preconcebida del pasado de la humanidad, del papel del pensamiento humano, de la experiencia, del empirismo positivo o de la inspiración metafísica en la formación de la ciencia dependerá sin duda alguna la solución que escojamos. El plan de una historia de las ciencias, al igual que sus conclusiones filosóficas, quedará así predeterminado. Desde el comienzo de nuestra investigación estaremos entonces encerrados en un círculo planteado por nosotros y del cual no podremos salir. Quizá sí, pero el escepticismo radical de quienes desdeñan la historia, de quienes creen que la historia es un espejo que le 
regresa al historiador su propio retrato espiritual, no podría ser sostenido a menos que, desde el primer contacto con los textos, el historiador detuviera su esfuerzo al proclamar inmediatamente que su juicio es definitivo, inamovible e irrevocable. Eso es lo que en efecto hacen las personas que no ven el pasado más recóndito, así como el pasado de ayer, de otra forma que a través del presente inmediato declarado perpetuo. Sin embargo, para el historiador que considera la historia como una realidad efectiva no podría ser de esa forma. El historiador no prejuzga los resultados de su trabajo cuando toma consciencia clara de las dificultades del mismo. En el curso de sus búsquedas se esfuerza constantemente por adentrarse en el pasado, lo cual le permite comprenderlo mejor, penetrar con mayor seguridad y mayor simpatía activa en el pensamiento creativo de antaño, al cual le infunde nueva vida, resucitándolo por un momento. Además, hay un factor personal, subjetivo como se dice con frecuencia, que es imposible de eliminar completamente; mejor reconocerlo abiertamente que negarlo a priori. Los historiadores, como todos los filósofos, como todos los sabios, y como todos los hombres tienen tendencias innatas, comportamientos del espíritu particulares e imperceptibles que no son todavía opiniones o sistemas, pero que pueden engendrar y que engendran opiniones y sistemas. Es usual, incluso, discutir la opinión o el sistema como si estuviera aislado, como si no hubiera nacido en un cerebro humano, como si no estuviera ordenado, imperiosamente ordenado, por ciertas tendencias primordiales; el historiador que tiene gran familiaridad con los textos de antaño lo sabe bien. Y en lugar de rebelarse contra la naturaleza de las cosas para obtener una objetividad espectral ubicada tanto fuera del mundo como de la ciencia, el historiador intenta encontrar o reconstituir por un momento, en sí mismo, los resortes profundos que subyacen a las obras objeto de su meditación. Cuando estudia los trabajos de los químicos cartesianos, de los químicos newtonianos, de los químicos stahlianos y de los químicos condillaquianos, que trabajaron e hicieron progresar su ciencia en el siglo XVIII, debe hacerse sucesivamente cartesiano, newtoniano, stahliano y condillaquiano. De este modo el historiador atrapa, o al menos intenta atrapar, las diferentes orientaciones de mentalidad que han sugerido las doctrinas, las hipótesis, las investigaciones experimentales de nuestros predecesores distantes; consigue comprender por completo o, por lo menos, toma la única vía que le permite comprender por completo los trabajos de diversas escuelas que han asociado sus investigaciones o que se han opuesto a ellas. En cuanto al factor personal, debido al carácter del historiador mismo, sin duda no será aniquilado. Lo que un historiador no habrá visto, otro historiador que aproveche la labor de su predecesor sabrá ponerlo en evidencia. Pero, ¿para qué insistir? Como todas las ciencias, la historia es una ciencia que se hace, que se continúa y que se renueva en la medida en que los 
progresos de la crítica y del análisis nos fuerzan a rectificar nuestras síntesis, las cuales jamás llegan a ser dogmáticas o definitivas.

Veamos las cosas un poco más de cerca. Para reconstituir el pensamiento de los hombres de ciencia de antaño (pienso en los químicos de los siglos XVII y XVIII), el historiador no tiene a su disposición, con frecuencia, más que los textos de esos hombres de ciencia en forma de libros, opúsculos o incluso disertaciones integradas en los trabajos de diversas academias o publicaciones científicas. Puede también consultar los textos anexos, las reseñas de las obras de química contenidas en esas publicaciones, los escritos de los filósofos, Regis o Berkeley, por ejemplo, que le dieron a la química una gran importancia, los estudios concernientes a las artes prácticas o las industriales, que como la metalurgia o la vidriería, estaban basadas en la química; las alusiones a la química contenidas en obras indiferentes a ella pero que pueden revelar el papel social de la química. A esto le podemos agregar ciertos laboratorios, o reproducciones de laboratorios que se estudian en los museos, por ejemplo en el Conservatorio de artes y oficios de París, las medallas conmemorativas, las estatuas y monumentos, los dibujos o las alegorías que ilustran un cierto número de publicaciones. Todas estas cosas tienen valores diversos. Pero para no perdernos en discusiones secundarias, sin embargo, les propongo limitarnos a los textos. No creo (aunque nunca se sabe) que ustedes se opondrán a mi opinión cuando les haya dicho que, a mi parecer, el objetivo último del historiador de las ciencias, como el del historiador de las letras, es lograr una comprensión completa de los textos que estudia. Para lograr dicho objetivo, se puede y se debe inspirar en los métodos serios y fecundos que han asegurado el éxito de la historia literaria, y que le han permitido verdaderamente resucitar el pasado. No les hablaré de estos métodos, y ustedes me concederán fácilmente que el historiador de las ciencias debe hacer un análisis filológico y crítico de los textos que estudia.

Es necesario reconocer, no obstante, que la obra científica que comparte ciertos rasgos con la obra puramente literaria, se distingue de ella por otras características que hacen difícil su acceso. No tengo necesidad de recordarles que las obras que el profesor de letras utiliza para nuestra cultura general con frecuencia tienen el privilegio de estar escritas, por así decirlo, como si estuvieran fuera del tiempo y del espacio; es decir, ellas se mantienen accesibles sin preparación alguna al lector inteligente siempre y en todo lugar. La única condición, por supuesto, es que el lector conozca la lengua del autor. Con objeto de no extraviarlos en una discusión sobre la preeminencia de las lenguas antiguas o modernas para la formación del espíritu, no hablaré más que de escritos franceses. Los estudiantes del liceo leen, por ejemplo, las tragedias de Racine, las comedias de Molière, los pensamientos de Pascal, las fábulas de La Fontaine, sin preocuparse por el "momento" de su aparición, como 
si estos tesoros del patrimonio de la humanidad no portaran el sello de una época. Confieso de inmediato que exagero para propósitos de esta exposición (pero no demasiado) cuando me refiero a la concepción habitual de las obras llamadas "clásicas". Sé que hay una buena parte de ilusión en estas concepciones y que esta ilusión, sobre la cual no nos detendremos a discutir hoy, ha sido disipada desde hace mucho tiempo por la crítica de Taine. De todos modos, sobre las obras que he mencionado, y muchas otras, se puede profundizar y meditar de muchas maneras para la educación, la instrucción y el placer del lector. Se puede reflexionar sobre aquello que uno lee en función de su propio pensamiento y sin preocuparse por el autor, con el sencillo fin de obtener de la lectura un beneficio intelectual o moral. Se puede leer también para conocer mejor al escritor, para adentrarse en su pensamiento y su sistema. Se puede leer, además, por muchos otros motivos. Finalmente, se pueden combinar diversas maneras de leer con el fin de comprender mejor el pasado, de comprender mejor el presente, de comprenderse mejor a sí mismo.

Que la obra científica de primer orden no sea completamente asimilable a la obra literaria de primer orden, es algo que no discutiré. No discutiré tampoco que la historia de la literatura general (en el sentido un tanto vago en que lo entienden los manuales de literatura) se encuentra con menos dificultades preliminares por superar que la historia de la literatura científica; sin embargo, me atrevo a adelantar que los escritos de los hombres de ciencia antiguos, incluyendo aquellos que están consagrados a teorías definitiva e irremediablemente caducas, no están del todo desprovistos de valor educativo y de poder sugestivo. Al igual que eso que llamamos las humanidades, estos escritos pueden contribuir a la cultura del espíritu. Pero, una vez más - y quizás me dirán ustedes, tanto como en las humanidades mismas-, estos escritos no entregan de manera inmediata su sustancia bienhechora a aquel que los hojea ya sea de manera precipitada, ya sea de manera perezosa; es necesario un esfuerzo asiduo de simpatía, exigido por su propio método, para que el historiador logre pensar los libros de los maestros de antaño como los habrían pensado los discípulos y los alumnos de esos maestros; para que se impregne, por ejemplo, de aquello que podía ser la ciencia que se hacía en el siglo XVII. Si logra aprehender la orientación del espíritu del hombre de ciencia cuya obra estudia, esta obra (aparte de eso que podríamos llamar su dimensión material, sobre la cual volveré más adelante), se le hará transparente y luminosa. Cuando lea a Van Helmont o a Lémery, se representará como telones de fondo los medios sociales o espirituales en los cuales estos químicos estaban sumergidos, pero también contra los cuales reaccionaban parcialmente. La obra de Van Helmont, por ejemplo, es un producto de la contrarreforma de comienzos del siglo XVII. Al atacar de manera violenta a la metafísica y la lógica de Aristóteles, al atacar a la astrología, él actúa como 
hombre de ciencia que libra a la química de un cierto número de supersticiones; pero también actúa como un cristiano que combate el regreso ofensivo del paganismo. Van Helmont se inspira en el Génesis, en una intuición intelectual que Dios envía de forma libre a aquellos de sus hijos que saben orar, trabajar con dolor y cumplir ciertas prácticas morales que mantienen su espíritu en un estado de receptividad. Los experimentos de laboratorio que ejecuta sin cesar, al enseñar directamente sobre el mundo, no hacen más que enseñar, y, además, necesitan ser aclarados, pues no enseñan por completo. Los experimentos se suman a la oración y a la meditación para disponer al químico a un estado en el que su alma pueda recibir una comunicación del pensamiento divino, y en ello consiste la comprensión auténtica, no en los argumentos lógicos que nos fuerzan a repetirnos de forma perpetua, es decir, sin jamás avanzar en el conocimiento de la naturaleza. Así el misticismo cristiano se alió al método experimental para renovar la ciencia de las reacciones materiales.

Pasemos ahora de la primera mitad del siglo XVII a la segunda mitad del mismo. Lleguemos a Lémery, quien piensa en clave cartesiana un mundo cartesiano. Admiremos al gran hombre que supo introducir definitivamente en la doctrina de su tiempo el espíritu claro y lúcido de su tiempo, así como supo imponer el estudio de la doctrina química al hombre honesto que no se enorgullece de nada pero que está al corriente de todo. Él eliminó de esta doctrina las analogías paracelsianas que encantaban el alegre vitalismo del renacimiento. Las esperanzas insensatas de los alquimistas concernientes a las transmutaciones de los metales se convirtieron en supersticiones absurdas y ruinosas. De manera general, Lémery expulsó de la ciencia todo lenguaje poético y alegórico. Pero no sólo en la teoría constatamos una revolución bienhechora: el arte de experimentar se modifica y se impregna de honestidad. Todo experimento de laboratorio que parezca truco de prestidigitación, que exija por lo menos un vuelco de mano difícil y sea mantenido en secreto, será considerado sospechoso, y Lémery no lo tendrá en cuenta. Él no quiere ser "peculiar en sus operaciones". Toda persona debe poder rehacer estas operaciones; su práctica no contiene ningún misterio, así como su teoría es ante todo "inteligible".

No me voy a entretener multiplicando los ejemplos. Sé muy bien, por otra parte, que al bosquejar muy rápidamente el telón de fondo intelectual y moral sobre el cual el historiador de las ciencias podrá poner de relieve las obras químicas, los descubrimientos prácticos, las sistematizaciones teóricas que ha aprehendido de Van Helmont o de Lémery, no les he hablado de las contribuciones definitivas de estos dos grandes hombres al conjunto del saber químico. Antes que nada, he buscado conocer la perspectiva científica (si es que se puede expresar así) de los hombres de ciencia que consagran su vida al perfeccionamiento o a la creación de 
una ciencia nueva; he buscado conocer la orientación de su mentalidad y eso, lo repito, es para mí de importancia primordial. De una generación a la siguiente, y a medida que la ciencia progresa, la perspectiva científica cambia; el problema que era central no interesa más a los investigadores, el pequeńo y curioso interrogante que intrigaba a los aficionados se convierte, en cambio, en problema central. Al tiempo que la perspectiva cambia, la orientación de la mentalidad se modifica. Al punto de vista que se encarnaba en cierto esfuerzo de sistematización le sucede una actitud por completo diferente, que engendra poco a poco por su movimiento mismo una nueva manera de comprender; y por supuesto, al ser la ciencia trabajo colectivo, los hombres de ciencia continúan la obra de sus predecesores, incluso si transforman, de manera brusca o por una evolución lenta, las doctrinas de estos predecesores. No porque al progresar la ciencia ceda a veces a la moda de la época y se impregne de los mismos principios que la opinión del momento, los conocimientos científicos cesan de ser acumulativos y progresivos, como lo ha dicho tan justamente el Señor Sarton. Y sin duda, los conocimientos no se acumulan como los inventarios de mercancías; se integran a monumentos doctrinales gracias a los hombres de ciencia. Pero la historia muestra que estos monumentos son remodelados constantemente, o reconstruidos sobre bases nuevas con los mismos materiales mezclados con otros nuevos.

No les tengo que hablar hoy de las revoluciones científicas, de sus significados con relación al progreso del espíritu humano o al progreso de nuestro conocimiento. Sin duda el historiador de la ciencia debe aportar a los filósofos que intentarán resolver los problemas resaltados aquí documentos cuya importancia será difícil de exagerar. Después de las obras magistrales de los seńores Brunschvicg y Meyerson, tan diferentes la una de la otra, pero que convergen en apoyar la epistemología en el estudio de las meditaciones científicas de antańo y de la actualidad, el historiador de las ciencias parece no tener más necesidad de abogados ante los filósofos para alegar en favor de su derecho a existir.

Una vez reconocida la importancia de la historia de las ciencias, no argumentaré entonces ante ustedes más que sobre el caso de la buena historia de las ciencias. No le impediré al historiador de las ciencias ser él mismo filósofo o partidario de tal o cual doctrina filosófica, le solicitaré simplemente que piense de manera histórica cuando haga historia. En el transcurso de su trabajo, no deberá inquietarse por saber si las conclusiones de su trabajo justificarán cierta concepción de la inteligencia o de la razón humana. No deberá encerrarse dentro de un esquema $a$ priori que le indique una concepción de antemano de la ciencia o de su historia. El acuerdo entre los hechos que el historiador estudia y la doctrina filosófica debe producirse por sí mismo sin trampas, sin solicitárselo a los hechos o a las doctrinas, 
sin empujarlos, en breve, sin arbitrariedades de ningún tipo. De esta manera, quizás, podremos buscar con alguna utilidad la solución al problema que el Sr. Lalande planteó a nuestras meditaciones. ¿Cuáles son las relaciones entre la razón constitutiva que en su tendencia permanente, esencial, es toda la razón humana, y la razón constituida que es el aspecto que presenta esta razón en cada momento del desarrollo de la humanidad?

Volvamos a la historia de las ciencias. Supongamos que el historiador dotado de buena voluntad y de agudeza espiritual haya por fin alcanzado a comprender la doctrina química como si fuera alumno de Van Helmont, de Stahl, de Lémery o de Macquer. ¿No encontrará ya ningún obstáculo y podrá proclamarse satisfecho por completo? ¡Qué desgracia! ¡Chocaría todavía con una dificultad actualmente no resuelta y que quizás no puede ser superada más que de forma parcial! Ya he señalado hace un momento "la dimensión material" y ahora debo hablar de ella.

Me voy a explicar una vez más con el ejemplo del químico. ${ }^{2}$ Este último entra en su laboratorio para trabajar de manera práctica y observar fenómenos provocados por su propio trabajo; puede mezclar ciertos cuerpos con ciertos otros y observar estas o aquellas reacciones materiales; puede realizar disoluciones, precipitaciones, evaporaciones, combustiones, calcinaciones y muchas otras cosas que haya previsto o no; consigna en sus escritos el resultado de sus experimentos empleando, naturalmente, el lenguaje de la teoría reinante; los explica, si puede, con la ayuda de esta teoría o los declara imprevistos y sorpresivos; con la ayuda de hipótesis auxiliares podrá tal vez dar cuenta de ellos. Ahora bien, es posible que, a pesar de que los textos sean precisos y claros, el historiador no capte por completo su significado real. Dados los reactivos presentes, le parece que el resultado del experimento habría debido ser otro. Y entonces se plantea un interrogante. Los cuerpos que el químico ha utilizado en sus operaciones de laboratorio, ¿son entonces los cuerpos que todavía llamamos con el mismo nombre? Es muy frecuente que sea necesario leer sulfuro de antimonio en vez de antimonio. En otras ocasiones, podemos preguntarnos si los reactivos son puros, homogéneos, o si contienen impurezas que modifican sus propiedades; el ácido de sal marina podría contener ácido vitriólico, lo mismo que el ácido nitroso e, incluso, ácido marino. En otras ocasiones, no se sabe y no hay modo alguno de saberlo. Así que cuando el historiador quiera hacerse contemporáneo de los autores cuya obra estudia, se verá en aprietos. Comprenderá las palabras, pero el suelo del laboratorio le parecerá sustraerse a sus pasos; el científico de antaño guardará con celo su secreto. Y como es deber siempre sacar

\footnotetext{
${ }^{2}$ Les recuerdo que Duhem en su bello libro sobre La teoría física, su objeto, su estructura estudió de manera similar el ejemplo de los físicos.
} 
ganancia de los fracasos, el historiador atisbará dos cosas que podrían habérsele escapado. En primer lugar, se dará cuenta de que los progresos de la química son mucho más grandes e importantes de lo que había creído en principio, pues los cuerpos puros colocados en frascos bien tapados y etiquetados con la fórmula química actual no nos fueron ofrecidos con gracia por la naturaleza; los reactivos son, podríamos decir casi sin exagerar, creados por la teoría. En todo caso, sin la ayuda de la teoría que orientó las investigaciones del químico, la ciencia jamás los habría obtenido y estudiado. El historiador se dará cuenta a continuación de que los hombres de ciencia de antaño no redactaron los resultados de sus investigaciones para facilitar el trabajo de los futuros historiadores de la ciencia. Tanto los químicos como los físicos y los naturalistas se empeñan en resolver un problema universal o particular que retiene su atención. Al dirigirse a sus contemporáneos se olvidan de insistir en las cosas que están fuera de discusión y que han recibido la adhesión unánime y espontánea de los hombres de ciencia. ¿Para qué extenderse sobre los fundamentos de la doctrina, que nadie sueña en discutir, porque todo el mundo los da por sentados? Que las cosas dejadas de lado se hayan vuelto primordiales para aquel que reflexiona muchos siglos después de la muerte de esos autores, que el historiador deba ante todo precisarlas para impregnarse de la orientación de la mentalidad de antaño, que para restablecerlas deba, por así decirlo, excavar por debajo del nivel en el que se asoma la doctrina, todo ello no hace nada fácil la reconstrucción del pasado. Y es por esta única razón que la historia de las letras y la historia de la filosofía se encuentran infinitamente más avanzadas que la historia de las ciencias. Ciertamente el historiador de las ciencias no carece de herramientas. Por ejemplo, en el siglo XVIII hubo polémicas violentas, que a veces degeneraron en disputas exaltadas, entre los químicos cartesianos, los químicos newtonianos, los químicos stahlianos, los químicos condillaquianos y los químicos que reclamaban un empirismo experimental integral; y estas polémicas y disputas nos aportan algunas lecciones preciosas acerca del estado del alma de los hombres de ciencia. Pero aquí también es necesario aconsejar prudencia. El sentido crítico del historiador está obligado a intervenir en la interpretación de textos que no se puede contentar con recoger y reunir. El historiador que quiere comprender el verdadero pensamiento de los hombres de ciencia no puede simplemente examinar los argumentos presentados por unos u otros, pues las doctrinas se establecen y se mantienen a causa de la satisfacción que le proporcionan a la inteligencia humana pero son atacadas o defendidas por las vías de menor resistencia. Por último, los datos de los problemas que los hombres de ciencia se esfuerzan por resolver no permanecen fijos y se modifican en la medida en que se descubren nuevos hechos o que se imaginan respuestas parciales a las preguntas realizadas. De una generación a la 
siguiente ocurre una suerte de desfase que hace variar tanto la orientación de la mentalidad como los datos teóricos y experimentales. Es así que para los químicos stahlianos, que habían advertido que toda calcinación de los metales es una combustión, pero que creían que los cuerpos al quemarse dejaban escapar su flogisto, el aumento del peso de los metales en el transcurso de una combustión se convertía en un enigma exasperante o divertido que había que explicar al margen de la teoría. Pero Lavoisier descubrió que los productos de la combustión del fósforo y del azufre pesan más que el fósforo o el azufre originales. Las calcinaciones de los metales no eran, pues, una excepción; al explicar por qué las cales de estaño o de plomo tienen una masa de materia más grande que el plomo o el estańo, Lavoisier no explica ya un hecho sorprendente y aislado, sino que da cuenta, al mismo tiempo, de todos los fenómenos de la combustión.

No voy a hacer más hincapié en esto. Debo observar, con todo, que no he hablado aquí más que del trabajo preliminar del historiador, pues hay todavía otro trabajo igualmente importante. Una vez que el historiador ha logrado hacerse contemporáneo de los hombres de ciencia cuyas teorías expone, apenas si ha cumplido con la mitad de su labor; le resta redactar su trabajo de tal manera que su lector logre adentrarse en las doctrinas de antaño, no sin ningún esfuerzo, o por lo menos uno mínimo. ¿Cómo alcanzar este objetivo? No se los diré hoy. Además, es al lector a quien le corresponde declarar si el historiador ha tenido éxito en su empresa de resurrección del pasado. 